\title{
Novel caries loci in children and adults implicated by genome-wide analysis of families
}

\author{
Manika Govil $^{1 * \dagger} \mathbb{D}$, Nandita Mukhopadhyay ${ }^{1 \dagger}$, Daniel E. Weeks ${ }^{2,3}$, Eleanor Feingold ${ }^{1,2,3}$, John R. Shaffer ${ }^{1,2}$, \\ Steven M. Levy ${ }^{4,5}$, Alexandre R. Vieira ${ }^{1,2}$, Rebecca L. Slayton ${ }^{6}$, Daniel W. McNeil ${ }^{7,8}$, Robert J. Weyant ${ }^{9}$, \\ Richard J. Crout ${ }^{10}$ and Mary L. Marazita ${ }^{1,2,11}$
}

\begin{abstract}
Background: Dental caries is a common chronic disease among children and adults alike, posing a substantial health burden. Caries is affected by multiple genetic and environmental factors, and prior studies have found that a substantial proportion of caries susceptibility is genetically inherited.

Methods: To identify such genetic factors, we conducted a genome-wide linkage scan in 464 extended families with 2616 individuals from lowa, Pennsylvania and West Virginia for three dental caries phenotypes: (1) PRIM: dichotomized as zero versus one or more affected primary teeth, (2) QTOT1: age-adjusted quantitative caries measure for both primary and permanent dentitions including pre-cavitated lesions, and (3) QTOT2: age-adjusted quantitative caries excluding pre-cavitated lesions. Genotyping was conducted for approximately 600,000 SNPs on an Illumina platform, pruned to 127,511 uncorrelated SNPs for the analyses reported here.
\end{abstract}

Results: Multipoint non-parametric linkage analyses generated peak LOD scores exceeding 2.0 for eight genomic regions, but no LOD scores above 3.0 were observed. The maximum LOD score for each of the three traits was 2.90 at 1q25.3 for PRIM, 2.38 at 6q25.3 for QTOT1, and 2.76 at 5q23.3 for QTOT2. Some overlap in linkage regions was observed among the phenotypes. Genes with a potential role in dental caries in the eight chromosomal regions include CACNA1E, LAMC2, ALMS1, STAMBP, GXYLT2, SLC12A2, MEGF10, TMEM181, ARID1B, and, as well as genes in several immune gene families. Our results are also concordant with previous findings from association analyses on chromosomes 11 and 19.

Conclusions: These multipoint linkage results provide evidence in favor of novel chromosomal regions, while also supporting earlier association findings for these data. Understanding the genetic etiology of dental caries will allow designing personalized treatment plans based on an individual's genetic risk of disease.

Keywords: Dental genetics, Dental public health, Permanent dentition caries, Primary dentition caries, Non-parametric linkage, Genome-wide linkage study

\section{Background}

Dental caries is one of the most common chronic diseases among children and adults alike. Childhood caries is associated with failure to thrive, and it can affect self-esteem and school performance [1]. For both children and adults, caries is associated with pain and loss of teeth, and caries

\footnotetext{
* Correspondence: manika.govil@gmail.com

${ }^{\dagger}$ Manika Govil and Nandita Mukhopadhyay contributed equally to this work.

${ }^{1}$ Center for Craniofacial and Dental Genetics, Department of Oral Biology,

School of Dental Medicine, University of Pittsburgh, Suite 500 Bridgeside

Point, 100 Technology Drive, Pittsburgh, PA 15219, USA

Full list of author information is available at the end of the article
}

may adversely impact growth and weight gain in children, as well as nutrition among adults, thereby negatively affecting quality of life.

Caries is known to have a genetic component. Detection of genetic factors is complicated by the fact that numerous diverse environmental factors influence the incidence and severity of this disease, including the microbiome, dietary habits, fluoride exposure, salivary factors and tooth structure.

Prior studies have shown that caries experience in humans is determined by genetic causes with heritability

(c) The Author(s). 2018 Open Access This article is distributed under the terms of the Creative Commons Attribution 4.0 International License (http://creativecommons.org/licenses/by/4.0/), which permits unrestricted use, distribution, and 
values between 20 and 60\% [2-7]. To date, there have also been numerous studies investigating association of dental caries with candidate genes or with whole-genome Single Nucleotide Polymorphism (SNP) panels $[8,9]$. The only previous genome-wide linkage study of caries was conducted using a panel of 392 microsatellite markers, on 46 extended Filipino families with 642 total individuals [10]. This study found suggestive linkage of low caries experience to chromosome regions 5q13.3, 14q11.2, and 13q27.1, and high caries experience to 13q31.1 and 14q24.3 However, results of previous studies have, in general, not been extensively replicated, possibly due to relatively small sample sizes $[8,9]$ and the enumeration of genetic factors is far from complete.

Our present study is the first to apply genome-wide multipoint linkage analysis to explore the genetic etiology of caries (whether in childhood or adulthood) using densely spaced SNPs on a population previously analyzed by genome-wide association. Genome-wide linkage analysis is a complementary strategy to genome-wide association analysis for gene-discovery. Whereas association identifies specific marker alleles correlated with the caries phenotype, linkage analysis strategies identify genomic regions shared between related individuals who show similar disease characteristics. The advantage of linkage analysis is that it makes full use of familial inheritance, is less sensitive to allelic heterogeneity, and, unlike association, can be used to detect rare disease-causing mutations. Furthermore, multipoint linkage utilizes genotypes from SNPs neighboring the test location, while association conducts tests at each location independently.

In this study, multipoint non-parametric linkage analysis was conducted, i.e., no assumptions were made with respect to the mode of inheritance of dental caries [11], and the analysis was, therefore, robust to uncertainty about the underlying genetic model. Empirical significance of the linkage signals was assessed across the genome by simulating multiple sets of genome-wide data such that the SNP genotypes were unlinked to caries status.

\section{Methods}

\section{Study subjects and genotype data}

The families and individuals included in this study are from western Pennsylvania, West Virginia, and Iowa. Subjects from Pennsylvania and West Virginia were ascertained through the Center for Oral Health Research in Appalachia (COHRA; [12]). Additional subjects from Pennsylvania were recruited under the University of Pittsburgh Dental Registry and DNA Repository (DRDR; [13]). Subjects from Iowa were recruited under two University of Iowa projects, the Iowa Fluoride Study (IFS; [14-17]) and Iowa Head Start (IHS; [18]). All subject recruitment and data collection was approved by site-specific Institutional Review Boards. Genotyping was conducted under the Gene Environment Association Studies Initiative (GENEVA) for approximately 600,000 SNPs on an Illumina platform (Human 610_Quadv1_B; Illumina, Inc., San Diego, CA, USA). All genotype and phenotype data is available on $\mathrm{dbGaP}$ (The database of Genotypes and Phenotypes; https:/www.ncbi.nlm.nih.gov/gap; accession number phs000095.v3.p1). Details on genotyping and quality control protocols are also presented on $\mathrm{dbGaP}$, or can be found in earlier studies $[19,20]$. Table 1 summarizes the different subsets of data in terms of the sample available for this study. This study utilizes complete families, in other words, non-genotyped and non-phenotyped individuals also contribute to various aspects of the analysis. Prior studies primarily utilized unrelated individuals for conducting association analysis. The starting study sample comprised a total of 4727 self-reported non-Hispanic white individuals, of which 437 were unrelated, 1674 were in 558 two-parent and single offspring (trio) families, and 2616 were in 464 non-trio families. Approximately $76 \%$ of individuals were genotyped (Table 1 ).

\section{Definition of dental caries phenotypes}

Caries scores were assessed on the COHRA, IFC, and IHS subjects in accordance with the COHRA study protocol [12]. For subjects in the DRDR study, we used caries scores abstracted from clinical records by dental students trained by Dr. Alexandre R. Vieira, who is a co-author on this manuscript.

Table 1 Starting sample size

\begin{tabular}{lllll}
\hline Site & \multicolumn{2}{l}{ Current study starting sample } & \\
\cline { 2 - 4 } & Unrelated & Trios $^{\mathrm{a}}$ (Individuals) & $\begin{array}{l}\text { Non-trio pedigrees } \\
\text { (Individuals) }\end{array}$ & Genotyped/Total \\
\hline COHRA & 29 & $162(486)$ & $452(2549)$ & $2209 / 3064$ \\
IFS & - & $394(1182)$ & $4(32)$ & $964 / 1214$ \\
IHS & 169 & $1(3)$ & $7(29)$ & $183 / 201$ \\
DRDR & 239 & $1(3)$ & $1(6)$ & $235 / 248$ \\
Total & 437 & $558(1674)$ & $464(2616)$ & $3591 / 4727$ \\
\hline
\end{tabular}

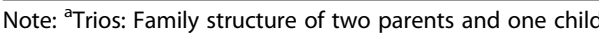

${ }^{\mathrm{b}}$ Non-trio pedigrees: Families with four or more members 
We defined three dental caries phenotypes, one based on primary dentition (PRIM), and two that combine primary and permanent dentitions (QTOT1, QTOT2). PRIM was coded as a binary primary dentition caries phenotype based on the count of decayed and/or filled primary teeth $(d f t)$ score. An individual with a $d f t$ score of 1 or more was designated as being affected. The primary teeth from all subjects with primary or mixed dentition were assessed for PRIM. These individuals included adults with over retained primary teeth. QTOT1, an age-adjusted quantitative caries phenotype, is based on the sum of the $d f t$ score (primary teeth) and $D_{1} M F T$ score (count of decayed, missing, and filled permanent teeth including white spot lesions). QTOT1 scores were generated by adjusting this raw sum for age and age-squared effects using locally fitted splines. Scores for 113 individuals below 2 years of age and 5 individuals above 60 years, were excluded from linkage analysis due to a very low caries experience in the $0-2$ years age group, and the presence of very few subjects above 60 years of age. Age-adjusted QTOT2, the second quantitative caries phenotype, is based on the sum of the $d f t$ score and the $D_{2} M F T$ score (count of decayed, missing, and filled permanent teeth excluding white spot lesions). Age-adjustment was performed as for the QTOT1 phenotype; and QTOT2 scores for 115 individuals between 0 and 2 years of age and 44 individuals above 60 years were set to missing.

\section{Data cleaning and preparation}

Genetic map positions were generated for all SNPs. These genetic markers were filtered based on genotyping rates and Hardy-Weinberg proportions. The SNPs were then pruned for linkage disequilibrium (LD). SNPs with residual LD were clustered into super-markers. The procedures used for filtering SNPs, map creation, and LD-based SNP pruning and clustering are described below.

\section{Genetic map creation}

The Genetic Map Interpolator (GMI) program, [21] was used to calculate genetic map positions for all SNPs. Sex-averaged map positions were created for SNPs on chromosomes 1-22, and female map positions were created for SNPs on the X chromosome. In the GMI program, the physical basepair (bp) position of each SNP per March 2006 Build NCBI36/hg18 was transformed to the corresponding centiMorgan (cM) scale genetic map distance based on interpolation into the Rutgers Combined Linkage-Physical Map [22].

\section{SNP filtering}

In addition to the quality control and cleaning steps detailed on dbGap, we filtered SNPs on the basis of low genotyping success rate and deviation from Hardy-Weinberg equilibrium (HWE) proportions using the software PLINK [23]. SNPs with genotyping success rates below 95\%, calculated using genotype data for all individuals, were excluded from analysis. Known genotypes of founders (i.e. those individuals in a family whose parents are not included in the study) and unrelated individuals were used to test SNPs for HWE proportions. The HWE proportions significance threshold was set at $10^{-5}$ for rejecting the null hypothesis of no deviation from HWE proportions.

\section{Linkage disequilibrium-based SNP pruning and clustering}

The genotyping panel available to this study was designed for genome-wide association analysis. When conducting linkage analysis on densely spaced SNP marker loci, the presence of substantial marker-to-marker LD is known to inflate linkage signals, especially if parental genotypes are missing [24]. In this study, LD was removed in two stages. First, the set of quality-filtered SNPs were pruned using PLINK such that the LD r ${ }^{2}$ (a measure of LD based on the square of the correlation coefficient between loci) value among remaining SNPs fell below 20\%. In PLINK, LD pruning consists of creating blocks of 50 consecutive SNPs followed by recursive removal of SNPs within blocks, until the LD r ${ }^{2}$ value among the remaining SNPs is below the desired threshold ( $20 \%$ in our case). Only the unrelated genotyped individuals in our data - pedigree founders and unrelated cases/controls - were used to calculate LD in this step. Any remaining LD was then accounted for using LD-based clustering in Merlin [25]. In clustering, each block of consecutive SNPs that shows an $\mathrm{r}^{2}$ value greater than a specified threshold (in our case $10 \%$ ), is analyzed collectively as a super-marker.

Table 2 summarizes the data processing steps undertaken to select SNPs for linkage analysis, and the samples that contributed information to specific parts of this data cleaning. After HWE filtering and LD-based pruning, 127,511 SNPs in low LD (pairwise $\mathrm{r}^{2} \leq 20 \%$ ) were retained. LD-based SNP clustering combined 92,495 SNPs into 20,634

Table 2 Sample for data cleaning

\begin{tabular}{ll}
\hline Procedure & Data \\
\hline Low genotype rate filtering (PLINK) & 3591 genotyped individuals \\
HWE testing (PLINK) & $\begin{array}{l}1839 \text { genotyped } \\
\text { (founders }^{\mathrm{a}}+\text { unrelated) }\end{array}$ \\
LD-based pruning (PLINK) & $\begin{array}{l}1839 \text { genotyped } \\
\text { (founders }^{\mathrm{a}}+\text { unrelated) }^{\mathrm{b}}\end{array}$ \\
$\begin{array}{l}1022 \text { families }^{-} \\
\text {LD-based clustering and }\end{array}$ & (trios + non-trio pedigrees) $^{c}$ \\
super-marker creation (Merlin) & 1022 families \\
Super-marker and SNP & (trios + non-trio pedigrees) $^{c}$ \\
allele frequency estimation (Merlin)
\end{tabular}

Note: a Founders: Individuals in a pedigree or trio whose parents are not included in the study. For example, both parents in a trio are founders. Also note that some of the larger multigenerational pedigrees may have more than two founders

${ }^{\mathrm{b}}$ The counts of individuals differs from totals provided in Table 1 since not all founders and unrelated individuals were genotyped for this study

'Trios: Family structure of two parents and one child; non-trio pedigrees: families with four or more members 
super-markers, leaving 35,016 SNPs to be analyzed individually. The average genetic map distance between the final set of markers (super-marker index and singleton SNPs) is approximately $0.07 \mathrm{cM}$ on the autosomes and $0.13 \mathrm{cM}$ on the X-chromosome. Super-marker and singleton SNP allele frequencies were generated as maximum likelihood estimates using Merlin. The SNP clustering and allele frequency estimation steps utilized 1022 informative families.

\section{Linkage analysis}

Table 3 summarizes the sample of individuals used within the linkage analysis for the three traits. In the table are presented the number of informative pedigrees, individuals, and phenotyped relative pairs by relationship type that were included in NPL and QT linkage. A total of 160 relative pairs were informative for PRIM NPL. For QT linkage, the corresponding informative relative pair counts were 1026 and 1038 for QTOT1 and QTOT2.

\section{Genome-wide linkage of PRIM}

In non-parametric linkage (NPL) analysis, affected individuals within each pedigree are examined to detect whether affected relatives share genomic regions identical-by-descent (IBD) more often than expected due to their relatedness alone. This IBD sharing is tested at locations along each chromosome. Genome-wide NPL analysis was carried out for the PRIM phenotype using the $\mathrm{S}_{\text {All }}$ statistic [26] as implemented in Merlin [25].

Table 3 Linkage analysis final sample

\begin{tabular}{llll}
\hline & PRIM & QTOT1 & QTOT2 \\
\hline Total Non-trio Pedigrees & 108 & 376 & 385 \\
COHRA & 106 & 372 & 373 \\
IFS & 1 & 4 & 4 \\
IHS & 1 & - & 7 \\
DRDR & - & - & 1 \\
Total Individuals & 687 & 2200 & 2243 \\
Phenotyped & 243 affected & 1738 & 1756 \\
Genotyped & 483 & 1582 & 1604 \\
Total informative relative pairs & \\
Median [Min, Max] pairs/pedigree & 160 & 1026 & 1038 \\
Sibling-pairs & $1[1-6]$ & $1[1-24]$ & $1[1-24]$ \\
Half-sibling pairs & 100 & 599 & 609 \\
Cousin pairs & 39 & 228 & 228 \\
Grandparent-grandchild & 21 & 73 & 73 \\
Avuncular & 0 & 28 & 28 \\
\hline
\end{tabular}

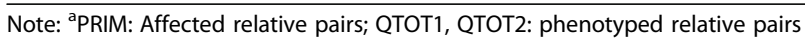

\section{Genome-wide linkage of QTOT1 and QTOT2}

The quantitative trait (QT) regression-based linkage method, Merlin-regress, [27] was utilized to carry out analyses of the two quantitative phenotypes across autosomes. The QT linkage method is based on regressing estimated IBD sharing between relative pairs on the squared sums and differences of their phenotypes. It does not handle $\mathrm{X}$-linked SNPs, hence the X chromosome was not analyzed for the two quantitative traits. Merlin-regress analyses required specification of a heritability parameter (set at $50 \%$ based on published estimates for DMFT) and sample-based means and variances for QTOT1 and QTOT2. All results, NPL and QT linkage, are reported as LOD (logarithm of the odds of linkage) scores.

\section{Empirical significance of observed linkage signals}

The most commonly used LOD score threshold of 3.0 used to test for significant linkage (Morton) was derived for parametric linkage analysis of a single locus on a binary trait phenotype. Subsequent research (e.g. those reviewed in [28]) that address newer linkage methods such as whole-genome analysis, affected-relative pairs and multipoint calculations are also based on assumptions on the study data, that are rarely true in real-life. Therefore, to correct for multiple testing, we carried out a simulation study to assess the genome-wide significance thresholds for the NPL and QT regression LOD scores. In general, for a null simulation, hundreds of simulated genetic data sets are generated and analyzed to produce an empirical distribution of LOD scores. Since this process would be prohibitively time consuming given the study data, we used an adaptive approach to generate null distributions. The replicate pool method, Pseudo [29] was used to derive the empirical null distribution of NPL scores for PRIM. An initial pool of 100 simulated genotype data sets was generated for this study using Merlin (simulate option) followed by the pseudo-simulation of 100,000 NPL genome-scans to create the empirical distribution of unlinked NPL LOD scores. Pseudo was not utilized for the quantitative data simulations since QT-regression does not produce pedigree-specific LOD scores. For QTOT1 and QTOT2, 5000 data sets each were simulated and analyzed using Merlin.

\section{Selection of linkage peak regions and etiologic genes}

For super-markers, the NPL and QT LOD scores correspond to the index (first) SNP of each cluster. In the linkage scan for each phenotype, maximizing markers in regions with $L O D$ score $\geq 2.0$ were identified as linkage peaks. A support interval of one LOD drop was used for exploring genes under selected linkage peaks. The one LOD drop support interval is the interval where the LOD score is within one unit of its maximum. 
Regions with LOD scores $\geq 1.0$ were identified for trait. Overlap of linkage signal among the three traits was determined based on overlap of peak support intervals or secondary peaks(s) of at least 1.0 LODs, lying within the primary peak support interval. In the event peaks for multiple phenotypes overlapped, the resulting support intervals were reduced to the region of overlap.

Genes within these support intervals were examined for a potential etiologic role in dental caries incidence. Genes identified as causal would include, for example, genes related to blood glucose levels, secretory function of the salivary glands, and host immune response. Proximity of genes to SNPs corresponding to linkage peaks was determined by physical map positions obtained from UCSC Genome Browser corresponding to the March 2006 (NCBI36/hg18) Assembly [30]. When no genes were identified as potentially contributing to caries risk, we instead listed the gene closest to the SNP with the maximum observed LOD.

\section{Comparison with prior published findings}

A systematic search of literature was conducted to compile caries risk-conferring genes and genomic regions from previous studies utilizing some portion of our data, as well as from studies of other populations. Physical positions for these genes and genomic regions were then mapped to our linkage scans. Linkage regions with a LOD score of 1.0 or greater have been reported as indicative of concordance or replication, as appropriate.

\section{Sensitivity analysis}

\section{Effect of variation in parameter values on NPL statistics}

For multifactorial diseases such as caries, the true underlying genetic model for disease is difficult to ascertain. In this study, model-free linkage methods were used to detect linkage. The QT methods are sensitive to the misspecification of the required programmatic input values. We conducted a sensitivity analysis for the heritability parameter (HP), since published literature provides a wide range of heritability values (40-60\%), and our work utilized $\mathrm{HP}=50 \%$. In the sensitivity analysis, $\mathrm{HP}$ values were set at 40, and 60\% for QTOT1 and QTOT2.

Mega2 [31] was used to re-format and create input files for all the software used in data cleaning, LD-based pruning and clustering, genetic map creation, linkage analysis and data simulation.

\section{Results}

\section{Study sample characteristics}

Figure 1 provides detailed information on the distribution of the three phenotypes, PRIM (panels A and B), QTOT1 (panels C, D, E and I), and QTOT2 (panels F, G, H and I). There were 287 individuals with known PRIM phenotypes (panel A), of which 243 individuals were affected for PRIM. Of these 243 subjects, 242 were 18 years or younger in age (panel B). Subjects with primary or mixed dentition included in the PRIM NPL analysis ranged from 15 months to 22.5 years of age, with a mean of 7.4 years. These subjects with primary dentition caries constitute mainly the youngest generation. The distribution of the raw caries index by decade, age-adjusted index by decade, and age-adjusted caries index within all phenotyped individuals compared to those between 2 and 60 years of age are shown for QTOT1 (panels C, D, and E) and QTOT2 (panels F, G, and H). The number of phenotyped individuals, range, mean and standard deviation are presented in panel I for both quantitative traits. A larger number of individuals were phenotyped for $D_{2} M F T$ as compared to $D_{1} M F T$ in this study. For QTOT1 and QTOT2, there were 2484, and 2868 phenotyped individuals in the 2-60 age range. Both of the age-adjusted phenotypes follow an approximately normal distribution, with a mean of zero. The QTOT1 and QTOT2 mean and standard deviations for the 2-60 age group were included as distribution parameters within quantitative trait linkage.

\section{Linkage analysis}

Figure 2 shows genome-wide LOD scores by SNP (or super-marker index SNP) for PRIM, QTOT1, and QTOT2. The empirical 5\% genome-wide significance level, indicated as a solid horizontal line, was 3.48, 3.61, and 3.76 for PRIM, QTOT1, and QTOT2, respectively. Overlapping LOD score peaks for multiple phenotypes were observed in a few regions.

\section{Highest LOD Score Regions}

Table 4 presents peak LOD scores and 1-LOD support intervals. The SNP (or index SNP) with the maximum LOD value in each peak is identified along with its genomic location. Regions with maximum $\mathrm{LOD} \geq 2.0$ are shown ordered by chromosome, along with secondary peak(s) of at least 1.0 LODs, if observed for other traits. The highest LOD scores by trait were 2.90 for PRIM, 2.38 for QTOT1, and 2.76 for QTOT2. Detailed results for all SNPs that lie within the support region for peaks reported in Table 4 with a LOD score of 2.0 or more are provided in supplementary material [see Additional File 1].

For each linkage peak, the table also reports the closest gene, if found, with a potential role in caries incidence. For two of these peaks, one on chromosome 2 (QTOT2; LOD 2.30) and the other on chromosome 3 (PRIM; LOD 2.50), no such etiologic genes were identified within the support intervals. In these intervals, genes BCL11A (60.538-60.634 Mb) and KAT2B (20.056$20.171 \mathrm{Mb}$ ) were found to be closest to the respective LOD score peak SNPs. The genes within linkage peak 


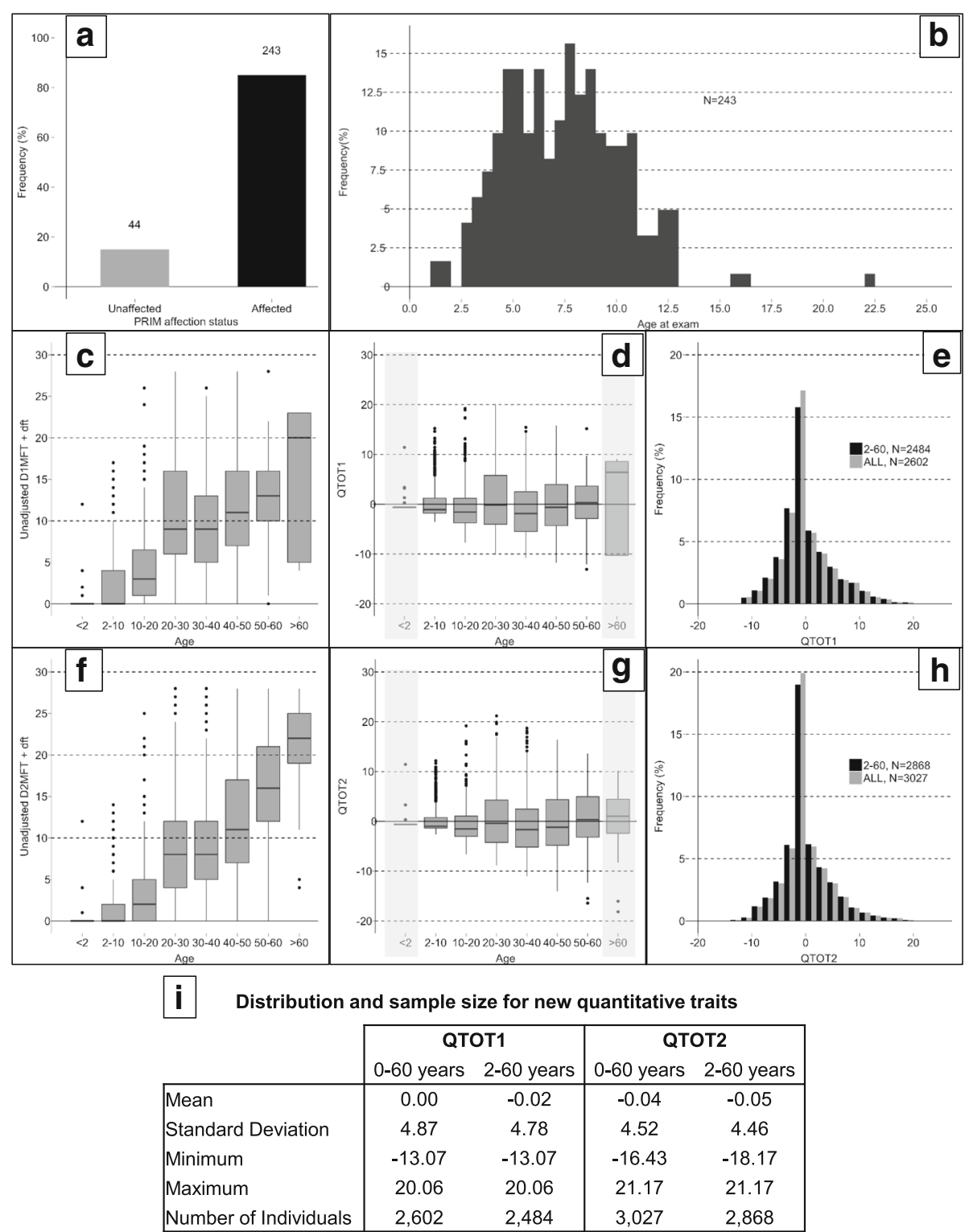

Fig. 1 Distribution of (a) PRIM by binary affection status, (b) age at exam of individuals categorized as PRIM affected, (c) raw dft $+D_{1}$ MFT (d) age-adjusted QTOT1, (e) age-adjusted QTOT1 for the full sample compared to the distribution for the 2-60 age group, (f) raw $d f t+D_{2} M F T,(\mathbf{g})$ age-adjusted QTOT2, (h) age-adjusted QTOT2 for the full sample compared to the distribution for the 2-60 age group, and (i) mean, standard deviation, range and sample size for QTOT1 and QTOT2; shaded areas in panels D and G indicate individuals below the age of 2 and above 60 years with phenotypes excluded from quantitative trait linkage analysis

regions that may play an etiologic role in dental caries are described in the sections below.

Chromosome 1 The highest LOD 2.90 across all three traits was observed on chromosome 1 for PRIM (Table 4). Under this peak, the CACNA1E (179.719-180.037 Mb) gene has been shown to be involved in glucose-evoked insulin secretion in mice [32]. Poor glycemic control has potential implications for increased caries risk in humans. Mutations in the LAMC2 (181.422-181.481 Mb) laminin gene are known to cause non-Herlitz form of junctional epidermolysis bullosa, which includes hypodontia and dental caries among its phenotypes [33].

Chromosome 2 The second QTOT2 peak on chromosome 2 includes the ALMS1 (73.466-73.691 Mb) gene. Mutations in this gene causes Alström syndrome, where gingivitis and discolored enamel are two clinical phenotypes [34]. Individuals with mutations in the STAMBP (73.910-73.944 Mb) gene have been reported to have cleft palate and facial dysmorphology [35]. 


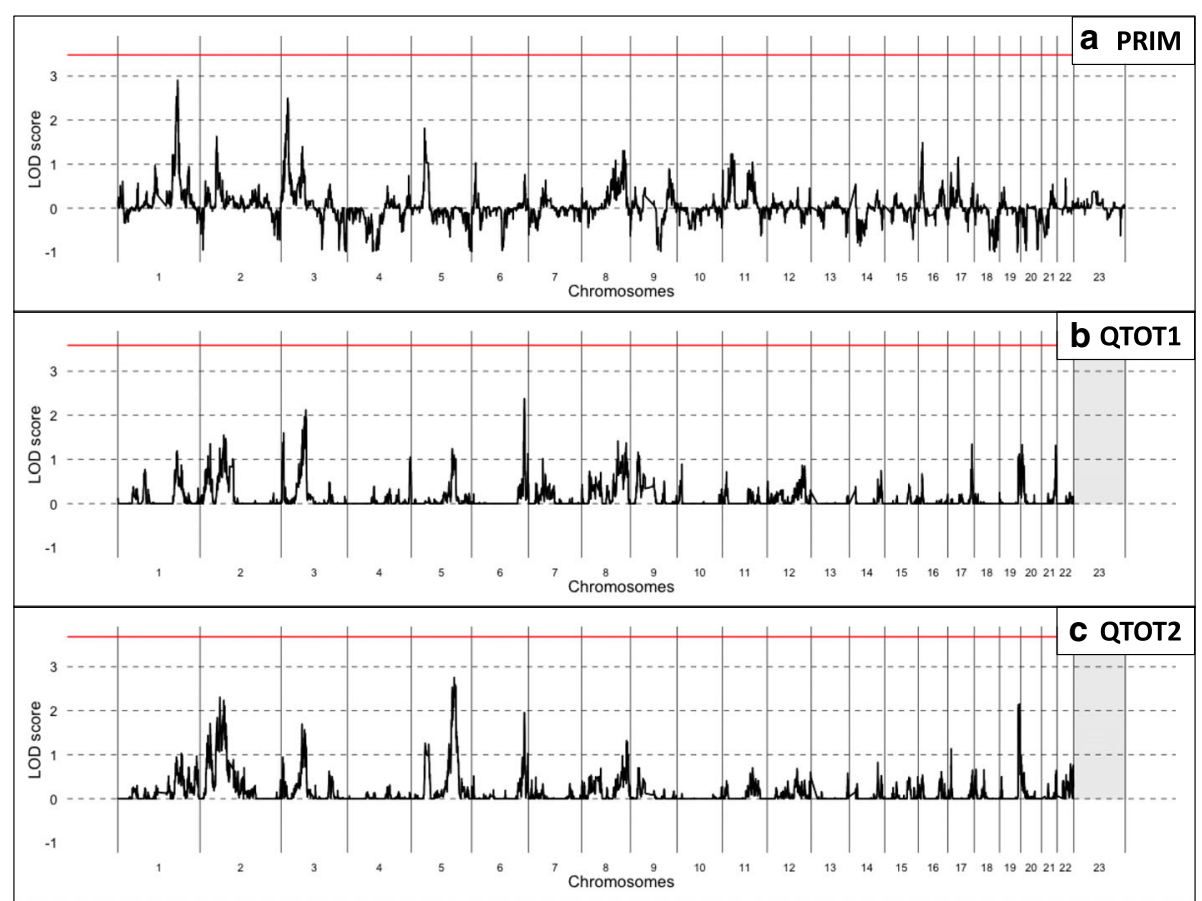

Fig. 2 Genome-wide LOD scores: (a) PRIM, (b) QTOT1, (c) QTOT2. PRIM results include the X chromosome. The empirical genome-wide 0.05 significance levels are indicated in each panel with a solid (red, online) horizontal line

Chromosome 3 The GXYLT2 (73.020-73.107 Mb) gene is located within the chromosome 3 QTOT1 peak. GXYLT2 acts on epidermal growth factor, which is expressed in human submandibular and parotid glands, and important for the maintenance of oroesophageal and gastric tissue.
Chromosome 5 The highest genome-wide quantitative trait LOD was observed for QTOT2. This QTOT2 peak contains the SLC12A2 (127.447-127.553 Mb) gene, whose protein product helps the movement of chloride ions in saliva, thereby assisting in salivary function. Also

Table 4 Linkage peaks in highest LOD score regions

\begin{tabular}{|c|c|c|c|c|c|c|c|}
\hline \multirow[t]{2}{*}{ Chr } & \multirow[t]{2}{*}{ Trait } & \multicolumn{3}{|l|}{ Peak $^{a}$} & \multicolumn{2}{|c|}{ Support Interval $(\mathrm{Mb})^{\mathrm{b}}$ for Peak with $L O D \geq 2$} & \multirow[t]{2}{*}{ Closest Genes within Support Interval $^{c}$} \\
\hline & & SNP & $\mathrm{bp}$ & LOD & Left & Right & \\
\hline 1 & QTOT1 & rs12096999 & $178,046,412$ & 1.19 & & & \multirow[t]{2}{*}{ CACNA1E; LAMC2 } \\
\hline 1 & PRIM & rs1281317 & $180,232,077$ & 2.90 & 174.78 & 182.03 & \\
\hline 2 & QTOT2 & rs7572396 & $59,893,993$ & 2.30 & 58.63 & 64.29 & $B C L 11 A^{\mathrm{d}}$ \\
\hline 2 & QTOT1 & rs13420242 & $71,117,276$ & 1.55 & & & \multirow[t]{2}{*}{ ALMS1; STAMBP } \\
\hline 2 & QTOT2 & rs831535 & $73,976,537$ & 2.10 & 65.23 & 79.72 & \\
\hline 3 & PRIM & rs9842115 & $20,378,197$ & 2.50 & 15.06 & 22.19 & $K A T 2 B^{d}$ \\
\hline 3 & QTOT1 & rs2044594 & $74,474,447$ & 2.12 & 67.65 & 76.08 & GXYLT2 \\
\hline 5 & QTOT1 & rs11748635 & $123,232,224$ & 1.24 & & & \multirow[t]{2}{*}{ SLC12A2; MEGF10; IL gene family } \\
\hline 5 & QTOT2 & rs6866597 & $128,905,516$ & 2.76 & 122.43 & 133.84 & \\
\hline 6 & QTOT1 & rs240642 & $158,117,314$ & 2.38 & 156.81 & 159.48 & \multirow[t]{2}{*}{ TMEM181; ARID1B } \\
\hline 6 & QTOT2 & rs9295289 & $158,387,494$ & 1.96 & & & \\
\hline 19 & QTOT1 & rs11084325 & $59,424,868$ & 1.12 & & & \multirow{2}{*}{$\begin{array}{l}\text { NLRP2; NLRP7; NLRP, KIR, and LILR } \\
\text { gene families }\end{array}$} \\
\hline 19 & QTOT2 & rs1671133 & $60,198,861$ & 2.15 & 59.42 & 61.47 & \\
\hline
\end{tabular}

Note: a Novel regions with LOD $\geq 2.00$, and secondary peaks $\geq 1.0$ observed for the other phenotypes; peak LOD $\geq 2.00$ shown in bold

${ }^{\mathrm{b}}$ Support interval for LOD $\geq 2.00 ; \mathrm{Mb}: 10^{6}$ (or 1 million) bp

'Genes with a potential role in caries incidence. If no such gene is identified, then the closest gene to the peak reported

dUnknown role in caries incidence; closest gene to the linkage peak SNP 
within the support interval are genes from the $I L$ family, which code for cytokines involved in blood production and immune system function. Defects in these genes result in autoimmune diseases and immune deficiency. A third gene, MEGF10 (126.654-126.825 Mb) has been implicated in MARDD (Myopathy, areflexia, respiratory distress, and dysphagia), with cleft palate as an associated phenotype [36].

Chromosome 6 The TMEM181 (158.877-158.976 Mb) gene under the QTOT1 linkage peak codes for a putative G-coupled protein receptor which mediates reaction to cytolethal distending toxins secreted by many pathogenic bacteria. ARID1B (157.141-157.572 Mb) mutations result in mental retardation along with minor teeth anomalies [37].

Chromosome 19 This region harbors several genes from the NLRP, KIR, and LILR immune gene families that code for various receptors within immune cells. NLRP2 (60.170-60.204 Mb), and NLRP7 (60.127$60.151 \mathrm{Mb}$ ) were closest to the peak.

\section{Comparison with previous relevant signals}

Table 5 shows regions reported by previous studies, where our current LOD score is 1.0 or greater. Two regions were found to contain genes reported in prior studies.

Chromosome 11 A PRIM LOD of 1.23 was observed $8500 \mathrm{bp}$ from the MPPED2 (30.338-30.558 Mb) gene. A suggestive association of primary teeth caries was reported by a previous study on 1305 children aged 3-12, some of whom are also part of this analysis (Shaffer et al., 2011). The phenotype was defined similarly to our PRIM phenotype.

Chromosome 19 A gene-set enrichment analysis study [38] reported an association of primary teeth caries to NLRP12 (58.989-59.019 Mb) in 1142 children aged 313 , a subset of whom are also included in our study. QTOT1 and QTOT2 LOD scores $\geq 1.0$ were observed $0.4-1.2 \mathrm{Mb}$ from this gene.

\section{Sensitivity analysis}

For each of the two quantitative traits, QTOT1 and QTOT2, Fig. 3, panels A, B, C, and D show the percentage deviation of LODs obtained using HPs of $40 \%$ or $60 \%$ from baseline LODs produced with an HP of $50 \%$. These deviations are plotted on the y-axis against the corresponding baseline LOD ( $x$-axis). The red points indicate SNPs for which baseline LODs of 2.0 or greater dropped below 2.0 when the HP value was changed. Conversely, the green points show SNP positions with baseline LODs below 2.0, which subsequently switched to a score of 2.0 or more with a change in HP. Percentage deviations where the baseline LODs were between 0 and 0.05 are not presented in panels A through D. Within this range, the change in LOD combined across $\mathrm{HP}=40 \%$ and $\mathrm{HP}=60 \%$ ranges from -0.04 to $0.11 \mathrm{LOD}$ for QTOT1. For QTOT2, the corresponding range is 0.05 to $0.1 \mathrm{LOD}$. Although in percentage terms they represent exponential changes as compared to the baseline, none of the deviations in the 0 to 0.5 baseline LOD score range result in the LOD score approaching significance. Panels E and F break down for each trait, the percentage of all SNPs that drop below-or exceed-the 2.0 LOD score threshold with a change in HP. For both traits, a decrease in HP to $40 \%$ results in a minimal percentage of SNPs changing status (be it an increase or decrease in LOD score). In contrast, SNPs with LOD scores of 2.0 or greater at HP 50\% are more likely to drop below the 2.0 LOD threshold when the HP is increased to $60 \%$.

Table 6 presents the change in QTOT1 and QTOT2 LOD scores due to a change in HP for only the linkage peaks reported in Table 4. All LOD score peaks, except for one, remain above 2.0 despite changes in HP.

\section{Discussion}

To our knowledge, this study was the first to apply genome-wide multipoint linkage analysis to explore the genetic etiology of caries using densely spaced SNPs.

We defined two new quantitative phenotypes which combine childhood and adulthood caries indices while also accounting for variability by age. The linkage findings in this study nominated genes on six chromosomes $(1,2$, $3,5,6$, and 19) with potential involvement in caries

Table 5 Linkage signals with LOD $\geq 1$ concordant with published findings

\begin{tabular}{|c|c|c|c|c|}
\hline \multirow[t]{2}{*}{ Study, Gene, phenotype } & \multicolumn{4}{|c|}{ Highest observed LOD Score } \\
\hline & SNP & $\mathrm{bp}$ & LOD & Trait \\
\hline Genome-wide association, MPPED2, dichotomized $d_{1} \mathrm{ft}^{\mathrm{a}}$ in US children aged 3-12 (Shaffer et al. 2011) & rs1447267 & $30,643,586$ & 1.23 & PRIM \\
\hline \multirow[t]{2}{*}{ Association with gene set enrichment, NLRP12, dichotomized $d_{1} \mathrm{ft}^{1}$ in children aged 3-12 (Wang et al. [38]) } & rs11084325 & $59,424,868$ & 1.12 & QTOT1 \\
\hline & rs1671133 & $60,198,861$ & 2.15 & QTOT2 \\
\hline
\end{tabular}

Note: aDichotomized $d_{1} f t$ as used in our study 


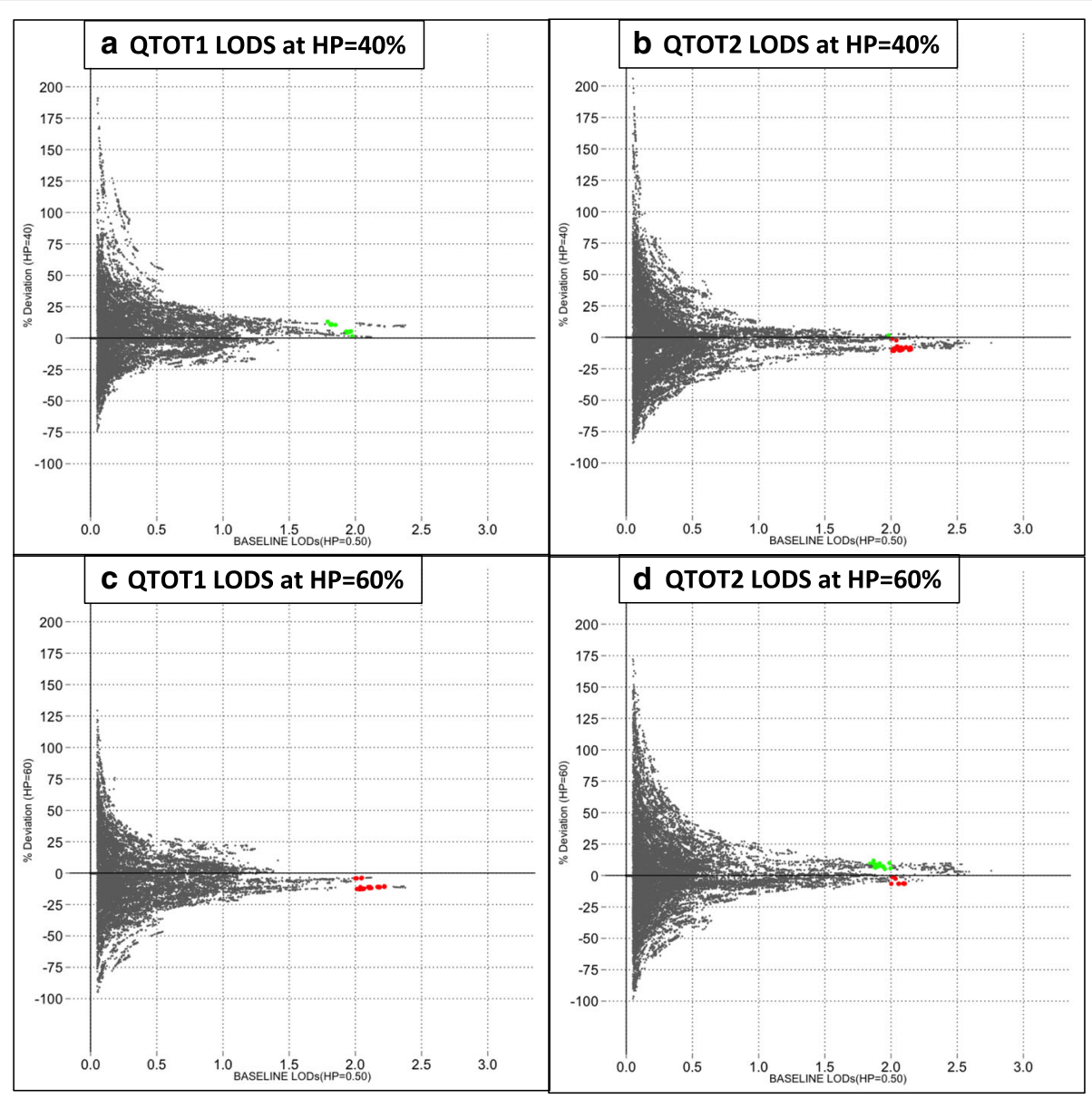

\begin{tabular}{|c|c|c|c|c|c|}
\hline \multicolumn{3}{|c|}{ e QTOT1 SNPs Switching LOD Threshold } & \multicolumn{3}{|c|}{ f QTOT2 SNPs Switching LOD Threshold } \\
\hline & $H P=40 \%$ & $H P=60 \%$ & & $H P=40 \%$ & $H P=60 \%$ \\
\hline HP $=50 \%$ LOD $<2$ switching $\geq 2$ & $0.026 \%$ & 0 & HP $=50 \%$ LOD $<2$ switching $\geq 2$ & $0.002 \%$ & $0.13 \%$ \\
\hline$H P=50 \%$ LOD $\geq 2$ switching $<2$ & 0 & $61.11 \%$ & HP $=50 \%$ LOD $\geq 2$ switching $<2$ & $0.06 \%$ & $5.39 \%$ \\
\hline
\end{tabular}

Fig. 3 Sensitivity of QT LOD score to changes in HP. Panels (a) and (c) are for QTOT1, and (b) and (d) are for QTOT2. In each scatterplot, the $x$-axis represents $L O D$ scores reported in this paper, using $H P=50 \%$. In panels $(\mathbf{a})$ and $(\mathbf{b})$, the $y$-axis represents $L O D$ scores for $H P=40 \%$. In panels (c) and (d), $y$-axis represents LOD scores calculated with HP $=60 \%$. Panels $E$ and $F$ show the proportion (\%) of SNPs switching from $L O D \leq 2.0$ to $L O D \geq 2.0$ for QTOT1 and QTOT2

Table 6 Comparison of reported peaks in HP sensitivity analysis

\begin{tabular}{llllll}
\hline Trait & Chr & SNP & $\mathrm{A}$ & $\mathrm{B}$ & $\mathrm{C}$ \\
& & & $\mathrm{HP}=50 \%^{\mathrm{a}}$ & $\mathrm{HP}=40 \%[\mathrm{~B}-\mathrm{A}]$ & $\mathrm{HP}=60 \%[\mathrm{C}-\mathrm{A}]$ \\
\hline QTOT1 & 3 & $\mathrm{rs} 2044594$ & 2.12 & $2.14[0.02]$ & $2.04[-0.08]$ \\
& 6 & $\mathrm{rs} 240642$ & 2.38 & $2.62[0.24]$ & $2.10[-0.28]$ \\
QTOT2 & 2 & $\mathrm{rs} 7572396$ & 2.30 & $2.20[-0.10]$ & $2.36[0.06]$ \\
& 2 & $\mathrm{rs} 831535$ & 2.10 & $2.16[0.06]$ & $2.00[-0.10]$ \\
& 5 & $\mathrm{rs6866597}$ & 2.76 & $2.64[-0.12]$ & $2.86[0.10]$ \\
& 19 & rs1671133 & 2.15 & $1.95[-0.20]$ & $2.28[0.13]$ \\
\hline
\end{tabular}

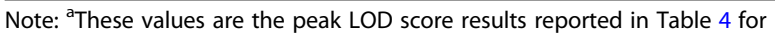
QTOT1 and QTOT2 etiology. Some of the genes are known to cause syndromes with a dental or oral phenotype, while others have a role to play in human immune and host defense response, blood glucose levels, and secretory function of the salivary glands all of which may have a potential impact on incidence of dental caries (see, for example, Carneiro et al. (2015) for the relationship between diabetes and dental caries). After a comprehensive review of the literature, we also detected linkage to regions on chromosomes 11 and 19 previously reported as associated to caries.

As expected, we do not recapitulate all findings from all prior association studies published by our group although this linkage study and the previous association studies utilized data from the same sources (i.e., 
COHRA, IHS, DRDR, IFS). As mentioned previously, linkage and association are complementary strategies for gene-discovery. In linkage, similarities and differences in pairs of phenotypes are modeled in terms of genetic similarity over related pairs from families. In association, this modeling is performed at the level of individuals. Our linkage uses multi-point analysis, i.e., the LOD score at any specific location is influenced by linkage at neighboring loci. Association generally uses a set of independent one-locus tests. Finally, as described in methods, this study differs from prior published work, both in the number, and the type (in terms of family composition) of individuals included in the analysis. Linkage utilizes family data and all related pairs (affected or phenotyped) within a pedigree whereas association generally is conducted on unrelated cases and controls, or at most parent-offspring trios.

The genotyping panel was designed for association analyses, and therefore, is far denser than a linkage SNP panel. Although dense bi-allelic SNP panels may allow extraction of more information, a concern for this study was existing linkage disequilibrium between SNPs. We pruned SNPs based on marker-to-marker LD, and then exploited any remaining LD among the pruned set to create clusters which served as polymorphic markers. Despite the pruning and clustering, our analysis was conducted on a much denser set of markers $(35,016$ SNPs and 20,634 super-markers) compared to a typical linkage panel with 6000 SNPs. The use of multi-allelic super-markers also had the potential of increasing power of linkage studies in such a setting.

Genome-wide significance for each phenotype was empirically assessed through a series of simulations, which provides an approximation of the true underlying distribution of a statistic since not all features of the data can be completely replicated. In an exploratory study, adhering strictly to genome-wide significance thresholds may be overly conservative. Furthermore, of the 4727 subjects, only 2616 contributed to the linkage analysis, providing a comparatively small number of relative pairs given the large sample size.

The sensitivity analysis conducted for the parameter HP explores the impact of parameter value selection on a model-free QT method. The results from this analysis indicated that the non-parametric quantitative trait linkage method, as implemented in Merlin, was robust to variation in HP, and that changing the HP parameter had a minimal impact on LOD scores. Even more importantly, the linkage peaks were insensitive to parameter misspecification.

Environmental factors are not accounted for in this study due to unavailability of such data on many of our subjects, which would have drastically reduced the cohort size. We also did not attempt to analyze gene-by-gene interaction. The available methods for detection of gene-gene interaction that are applicable to our study design are computationally complex, thus making whole-genome interaction analysis beyond the scope of the current work (e.g. see the review of the various classes of interaction detection methods by Li [39]).

\section{Conclusions}

This study presents two new quantitative measures for dental caries which combine both the primary and permanent dentition, while adjusting for age effects. Genes identified in peak linkage regions underline the importance of exploring potential relationships between caries and other traits. We did not include environmental factors in this study. The interaction between putative caries risk conferring genes and factors including fluoride exposure, dietary habits, and the microbiome need to be investigated, as do interactions between the genes themselves. From a clinical perspective, individuals would be at an elevated lifetime risk of developing caries in both primary and permanent dentition, given increased genetic susceptibility. Understanding the genetic etiology of dental caries will allow health providers to design personalized treatment plans based on an individual's genetic risk of disease.

\section{Additional file}

Additional file 1: SNPs within support regions of reported peaks. Detailed results for all SNPs that lie within the support region for peaks with a LOD score of 2.0 or more, as summarized in Table 4 of the main paper. (DOCX $97 \mathrm{~kb}$ )

\section{Abbreviations}

bp: basepair; cM: centiMorgan; COHRA: Center for Oral Health Research in Appalachia; $D_{1}$ MFT: Count of decayed, missing, and filled permanent teeth including white spot lesions; D2MFT: Count of decayed, missing, and filled permanent teeth excluding white spot lesions; dbGaP: The database of Genotypes and Phenotypes; dft: Count of decayed and/or filled primary teeth (dft) score; DRDR: University of Pittsburgh Dental Registry and DNA Repository; GENEVA: Gene Environment Association Studies Initiative; GMI: Genetic Map Interpolator; HP: Heritability Parameter; HWE: HardyWeinberg Equilibrium; IBD: Identical-By-Descent; IFS: Iowa Fluoride Study; IHS: lowa Head Start; LD: Linkage Disequilibrium; LOD: Logarithm of the odds of linkage; MARDD: Myopathy, areflexia, respiratory distress, and dysphagia; Mb: $10^{6}$ (or 1 million) bp; NPL: Non-Parametric Linkage; PRIM: New binary dental caries phenotype based on dft; QT: Quantitative Trait; QTOT1: New age-adjusted quantitative caries phenotype based on the sum of the $\mathrm{dft}$ score and D MFT score; QTOT2: New age-adjusted quantitative caries phenotype based on the sum of the $\mathrm{dft}$ score and $\mathrm{D}_{2}$ MFT score; $r^{2}$ : A measure of LD based on the square of the correlation coefficient between loci); SNP: Single Nucleotide Polymorphism

\section{Acknowledgements}

Many thanks are due to the study participants, and also to the dedicated research staff at all of the research sites, without whom these studies would be impossible.

Support for analyses was provided by the National Institute of Dental and Craniofacial Research (NIDCR) as a Pathway to Independence Award (R00-DE018085). Funding for genotyping was provided by NIDCR as part of the trans-NIH Genes, Environment and Health Initiative [GEI] (U01-DE018903). Genotyping was done by the Johns Hopkins University (JHU) Center for Inherited Disease Research (CIDR), with funding from the NIDCR, contract number HHSN268200782096C. Assistance with phenotype harmonization 
and genotype cleaning, as well as with general study coordination, was provided by the GENEVA Coordinating Center (U01-HG004446) and by NCBI. Data and samples were provided by (1) the Center for Oral Health Research in Appalachia (a collaboration of the University of Pittsburgh and West Virginia University funded by NIDCR R01-DE 014899); (2) the lowa Fluoride Study and the lowa Bone Development Study, funded by NIDCR R01DE09551and R01-DE12101, respectively); (3) the lowa Comprehensive Program to Investigate Craniofacial and Dental Anomalies (funded by NIDCR, P60-DE-13076. The Dental Registry and DNA Repository project is supported by the University of Pittsburgh School of Dental Medicine. All analyses were conducted on the Centos 276-node cluster, Indy, also supported by the School of Dental Medicine at the University of Pittsburgh.

\section{Funding}

National Institute of Dental and Craniofacial Research (NIDCR), Award Numbers: R00-DE018085, U01-DE018903, HHSN268200782096C, R01-DE 014899, R01-DE09551, R01-DE12101, P60-DE-13076.

GENEVA Coordinating Center Award Number: U01-HG004446. Neither NIDCR nor the GENEVA Coordinating Center played a role in the design of the study and collection, analysis, and interpretation of data and in writing the manuscript.

\section{Availability of data and materials}

All genotype and phenotype data for the datasets analyzed during the current study are available on dbGaP repository, [https://www.ncbi.nlm.nih.gov/gap; accession number phs000095.v3.p1]

\section{Authors' contributions}

MG, NM, DEW, EF, MLM conceived and designed this study; NM and MG analyzed the data; MG and NM wrote the manuscript; SML, ARV, RLS, DWM RJW, RJC, and MLM acquired the data; NM, MG, managed, cleaned and quality checked the data for linkage; MG, NM, DEW, EF, JRS, and MLM interpreted the results. All authors read and approved the final manuscript.

\section{Ethics approval and consent to participate}

All subject recruitment and data collection was approved by the site-specific Institutional Review Boards listed below. At each site, written consent to participate was obtained from all study subjects.

COHRA participants: University of Pittsburgh Institutional Review Board and West Virginia University Institutional Review Board.

IHS participants: University of lowa Institutional Review Board.

IFS participants: University of lowa Institutional Review Board.

DRDR participants: University of Pittsburgh Institutional Review Board.

\section{Competing interests}

The authors declare that they have no competing interests.

\section{Publisher's Note}

Springer Nature remains neutral with regard to jurisdictional claims in published maps and institutional affiliations.

\section{Author details}

${ }^{1}$ Center for Craniofacial and Dental Genetics, Department of Oral Biology, School of Dental Medicine, University of Pittsburgh, Suite 500 Bridgeside Point, 100 Technology Drive, Pittsburgh, PA 15219, USA. ²Department of Human Genetics, Graduate School of Public Health, University of Pittsburgh, Pittsburgh, PA, USA. ${ }^{3}$ Department of Biostatistics, Graduate School of Public Health, University of Pittsburgh, Pittsburgh, PA, USA. ${ }^{4}$ Department of Preventive and Community Dentistry, University of lowa College of Dentistry, lowa City, IA, USA. ${ }^{5}$ Department of Epidemiology, University of lowa College of Public Health, lowa City, IA, USA. ${ }^{6}$ Department of Pediatric Dentistry, School of Dentistry, University of Washington, Seattle, WA, USA. ${ }^{7}$ Denta Practice and Rural Health, West Virginia University School of Dentistry, Morgantown, WV, USA. ${ }^{8}$ Department of Psychology, Eberly College of Arts and Sciences, West Virginia University, Morgantown, WV, USA. ${ }^{9}$ Department of Dental Public Health and Information Management, School of Dental Medicine, University of Pittsburgh, Pittsburgh, PA, USA. ${ }^{10}$ Department of Periodontics, West Virginia University School of Dentistry, Morgantown, WV USA. ${ }^{11}$ Clinical and Translational Science Institute, and Department of Psychiatry, School of Medicine, University of Pittsburgh, Pittsburgh, PA, USA.
Received: 3 March 2017 Accepted: 22 May 2018

Published online: 01 June 2018

\section{References}

1. Chou R, Cantor A, Zakher B, Mitchell JP, Pappas M: Prevention of Dental Caries in Children Younger Than 5 Years Old: Systematic Review to Update the U.S. Preventive Services Task Force Recommendation. Evidence Synthesis No. 104. ; 2014

2. Boraas JC, Messer LB, Till MJ. A genetic contribution to dental caries, occlusion, and morphology as demonstrated by twins reared apart. J Dent Res. 1988:67(9):1150-5.

3. Bretz WA, Corby PM, Schork NJ, Robinson MT, Coelho M, Costa S, Melo Filho MR, Weyant RJ, Hart TC. Longitudinal analysis of heritability for dental caries traits. J Dent Res. 2005;84(11):1047-51.

4. Morrison J, Laurie CC, Marazita ML, Sanders AE, Offenbacher S, Salazar CR, Conomos MP, Thornton T, Jain D, Laurie CA, et al. Genome-wide association study of dental caries in the Hispanic communities health study/study of Latinos (HCHS/SOL). Hum Mol Genet. 2016;25(4):807-16.

5. Shaffer JR, Wang X, Desensi RS, Wendell S, Weyant RJ, Cuenco KT, Crout R, McNeil DW, Marazita ML. Genetic susceptibility to dental caries on pit and fissure and smooth surfaces. Caries Res. 2012;46(1):38-46.

6. Shaffer JR, Wang X, McNeil DW, Weyant RJ, Crout R, Marazita ML. Genetic susceptibility to dental caries differs between the sexes: a family-based study. Caries Res. 2015;49(2):133-40.

7. Wang X-J, Shaffer JR, Weyant RJ, Cuenco KT, DeSensi RH, Crout RJ, McNeil DW, Marazita ML. Genes and their effects on dental caries may differ between primary and permanent dentitions. Caries Res. 2010;44(3):277-84.

8. Vieira AR, Modesto A, Marazita ML. Caries: review of human genetics research. Caries Res. 2014;48(5):491-506.

9. Opal S, Garg S, Jain J, Walia I. Genetic factors affecting dental caries risk. Aust Dent J. 2015;60(1):2-11.

10. Vieira AR, Marazita ML, Goldstein-McHenry T. Genome-wide scan finds suggestive caries loci. J Dent Res. 2008;87(5):435-9.

11. Kruglyak L, Daly MJ, Reeve-Daly MP, Lander ES. Parametric and nonparametric linkage analysis: a unified multipoint approach. Am J Hum Genet. 1996:58(6):1347-63

12. Polk DE, Weyant RJ, Crout RJ, McNeil DW, Tarter RE, Thomas JG, Marazita ML. Study protocol of the Center for Oral Health Research in Appalachia (COHRA) etiology study. BMC oral health. 2008:8:18.

13. Vieira AR, Hilands KM, Braun TW. Saving more teeth-a case for personalized care. J Pers Med. 2015;5(1):30-5.

14. Franzman MR, Levy SM, Warren JJ, Broffitt B: Tooth-brushing and dentifrice use among children ages 6 to 60 months. Pediatr Dent 2004, 26(1):87-92.

15. Levy SM, Warren JJ, Broffitt B, Hillis SL, Kanellis MJ. Fluoride, beverages and dental caries in the primary dentition. Caries Res. 2003;37(3):157-65.

16. Levy SM, Warren JJ, Davis CS, Kirchner HL, Kanellis MJ, Wefel JS. Patterns of fluoride intake from birth to 36 months. J Public Health Dent. 2001;61(2):70-

17. Marshall TA, Levy SM, Broffitt B, Warren JJ, Eichenberger-Gilmore JM, Burns TL, Stumbo PJ. Dental caries and beverage consumption in young children. Pediatrics. 2003;112(3 Pt 1):e184-91.

18. Slayton RL, Cooper ME, Marazita ML. Tuftelin, mutans streptococci, and dental caries susceptibility. J Dent Res. 2005;84(8):711-4.

19. Wang X, Shaffer JR, Zeng Z, Begum F, Vieira AR, Noel J, Anjomshoaa I, Cuenco KT, Lee MK, Beck J, et al. Genome-wide association scan of dental caries in the permanent dentition. BMC oral health. 2012;12:57.

20. Shaffer JR, Wang X, Feingold E, Lee M, Begum F, Weeks DE, Cuenco KT, Barmada MM, Wendell SK, Crosslin DR, et al. Genome-wide association scan for childhood caries implicates novel genes. J Dent Res. 2011;90(12):1457-62.

21. Genetic Map Interpolator [https://watson.hgen.pitt.edu/register/].

22. Matise TC, Chen F, Chen W, De La Vega FM, Hansen M, He C, Hyland FC, Kennedy GC, Kong X, Murray SS, et al. A second-generation combined linkage physical map of the human genome. Genome Res. 2007;17(12): 1783-6.

23. Purcell S, Neale B, Todd-Brown K, Thomas L, Ferreira MA, Bender D, Maller J, Sklar P, de Bakker PI, Daly MJ, et al. PLINK: a tool set for whole-genome association and population-based linkage analyses. Am J Hum Genet. 2007; 81(3):559-75.

24. Levinson DF, Holmans P. The effect of linkage disequilibrium on linkage analysis of incomplete pedigrees. BMC Genet. 2005;6(Suppl 1):S6. 
25. Abecasis GR, Cherny SS, Cookson WO, Cardon LR. Merlin-rapid analysis of dense genetic maps using sparse gene flow trees. Nat Genet. 2002;30(1):97-101.

26. Whittemore AS, Halpern J. A class of tests for linkage using affected pedigree members. Biometrics. 1994;50(1):118-27.

27. Sham PC, Purcell S, Cherny SS, Abecasis GR. Powerful regression-based quantitative-trait linkage analysis of general pedigrees. Am J Hum Genet. 2002;71(2):238-53

28. Nyholt DR. All LODs are not created equal. Am J Hum Genet. 2000;67(2): 282-8.

29. Wigginton JE, Abecasis GR. An evaluation of the replicate pool method: quick estimation of genome-wide linkage peak p-values. Genet Epidemiol. 2006;30(4):320-32.

30. UCSC Genome Browser [http://genome.ucsc.edu]

31. Baron RV, Kollar C, Mukhopadhyay N, Weeks DE. Megaz: validated datareformatting for linkage and association analyses. Source Code Biol Med. 2014;9(1):26.

32. Jing X, Li DQ, Olofsson CS, Salehi A, Surve W, Caballero J, Ivarsson R, Lundquist I, Pereverzev A, Schneider T, et al. CaV2.3 calcium channels control second-phase insulin release. J Clin Invest. 2005;115(1):146-54.

33. Bircher AJ, Lang-Muritano M, Pfaltz M, Bruckner-Tuderman L. Epidermolysis bullosa junctionalis progressiva in three siblings. Br J Dermatol. 1993;128(4): 429-35.

34. Ozgul RK, Satman I, Collin GB, Hinman EG, Marshall JD, Kocaman O, Tutuncu Y, Yilmaz T, Naggert JK. Molecular analysis and long-term clinical evaluation of three siblings with Alstrom syndrome. Clin Genet. 2007;72(4):351-6.

35. Carter MT, Geraghty MT, De La Cruz L, Reichard RR, Boccuto L, Schwartz CE, Clericuzio CL. A new syndrome with multiple capillary malformations, intractable seizures, and brain and limb anomalies. Am J Med Genet A. 2011;155a(2):301-6.

36. Hartley L, Kinali M, Knight R, Mercuri E, Hubner C, Bertini E, Manzur AY, Jimenez-Mallebrera C, Sewry CA, Muntoni F. A congenital myopathy with diaphragmatic weakness not linked to the SMARD1 locus. Neuromuscul Disord. 2007:17(2):174-9.

37. Hoyer J, Ekici AB, Endele S, Popp B, Zweier C, Wiesener A, Wohlleber E, Dufke A, Rossier E, Petsch C, et al. Haploinsufficiency of ARID1B, a member of the SWI/SNF-a chromatin-remodeling complex, is a frequent cause of intellectual disability. Am J Hum Genet. 2012;90(3):565-72.

38. Wang Q, Jia P, Cuenco KT, Zeng Z, Feingold E, Marazita ML, Wang L, Zhao $Z$. Association signals unveiled by a comprehensive gene set enrichment analysis of dental caries genome-wide association studies. PLoS One. 2013; 8(8):e72653.

39. Li C: Detecting gene-gene interaction in linkage analysis. Current protocols in human genetics 2005, Chapter 1:Unit 1.15 .

\section{Ready to submit your research? Choose BMC and benefit from:}

- fast, convenient online submission

- thorough peer review by experienced researchers in your field

- rapid publication on acceptance

- support for research data, including large and complex data types

- gold Open Access which fosters wider collaboration and increased citations

- maximum visibility for your research: over $100 \mathrm{M}$ website views per year

At BMC, research is always in progress.

Learn more biomedcentral.com/submissions 